\title{
Methamphetamine Exposure Combined with HIV-I Disease or gp 120 Expression: Comparison of Learning and Executive Functions in Humans and Mice
}

\author{
James P Kesby', Robert K Heaton', Jared W Young ',2, Anya Umlauf', Steven P Woods', Scott L Letendre', \\ Athina Markou', Igor Grant' and Svetlana Semenova*,I \\ 'Department of Psychiatry, School of Medicine, University of California San Diego, La Jolla, CA, USA; ${ }^{2}$ Research Service, VA San Diego Healthcare \\ System, San Diego, CA, USA
}

\begin{abstract}
Methamphetamine dependence is a common comorbid condition among people living with HIV, and may exacerbate HIV-associated neurocognitive disorders. Animal models of neuroAIDS suggest that the gp I 20 protein may also cause cognitive impairment. The present work evaluated the separate and combined effects of HIV/gpl20 and methamphetamine on learning and executive functions in both humans and transgenic mice. Human participants were grouped by HIV serostatus (HIV+ or HIV - ) and lifetime methamphetamine dependence (METH+ or METH-). A neurocognitive test battery included domain-specific assessments of learning and executive functions. Mice (gpl20+ and gpl20-) were exposed to either a methamphetamine binge (METH+) or saline $(\mathrm{METH}-$ ), then tested in the attentional-set-shifting task to assess learning and executive functions. In humans, HIV status was associated with significant impairments in learning, but less so for executive functions. The frequency of learning impairments varied between groups, with the greatest impairment observed in the HIV+/METH+ group. In mice, gp I 20 expression was associated with impairments in learning but not reversal learning (executive component). The greatest proportion of mice that failed to complete the task was observed in the gp 120 +/METH+ group, suggesting greater learning impairments. Our cross-species study demonstrated that HIV in humans and gp I 20 in mice impaired learning, and that a history of methamphetamine exposure increased the susceptibility to HIV-associated neurocognitive deficits in both species. Finally, the similar pattern of results in both species suggest that the gp I 20 protein may contribute to HIV-associated learning deficits in humans.

Neuropsychopharmacology (2015) 40, I899-1909; doi:I0.I038/npp.2015.39; published online 25 February 2015
\end{abstract}

\section{INTRODUCTION}

In the era of combination antiretroviral therapy, over $35 \%$ of people with HIV (HIV+) exhibit neurocognitive impairments, with up to $10 \%$ showing moderate to severe impairment (consistent with HIV-associated dementia; Heaton et al, 2011). HIV-associated cognitive deficits include impairments in learning, executive functions, working memory, recall (episodic memory), processing speed, and motor skills (Heaton et al, 1995; Lindl et al, 2010). Both HIV disease progression and HIV-associated cognitive deficits may be exacerbated by comorbid drug use (Carrico, 2011). Methamphetamine dependence, in particular, is a common comorbidity of HIV infection (Marquez et al, 2009) and has been linked to increased neurocognitive impairments in

*Correspondence: Dr S Semenova, Department of Psychiatry, School of Medicine, University of California San Diego, 9500 Gilman Drive, M/C 0603, La Jolla, CA 92093, USA, Tel: +858 534 1528, Fax: +858 534 9917, E mail: ssemenova@ucsd.edu

Received I5 October 2014; revised I5 January 2015; accepted 27 January 2015; accepted article preview online 5 February 2015
HIV+ individuals (Rippeth et al, 2004; Gupta et al, 2011). Considering that HIV disease is associated with pronounced impairments in learning (Heaton et al, 2011) and a wide variety of executive functions (Heaton et al, 2011; Weber et al, 2013), we hypothesized that deficits in these two cognitive domains may be exacerbated in HIV+ methamphetamine users because of common mechanisms of neuropathology.

The combination of HIV and methamphetamine may lead to greater declines in cognitive performance by preferentially targeting the basal ganglia. Specifically, HIV-induced decreases in caudate/basal ganglia volume (Aylward et al, 1993; Kieburtz et al, 1996) and dopamine levels (Kumar et al, 2009) have been associated with poorer cognitive performance (Hestad et al, 1993; Kieburtz et al, 1996). Similarly, methamphetamine exposure causes neurotoxicity in mesolimbic and mesocortical brain regions (Mark et al, 2004). Individuals who are both methamphetamine dependent and HIV+ show exacerbated neuronal injury (Chang et al, 2005) and cortical interneuron loss (Langford et al, 2003; Chana et al, 2006), which may also contribute to impaired cognitive performance. The role of specific HIV-associated products 
that could contribute to the interactive effects of methamphetamine and HIV on learning and executive functions is less understood.

Accumulating evidence indicates that HIV-encoded proteins, such as gp120, contribute to neuronal dysfunction in the basal ganglia (Purohit et al, 2011). Moreover, the presence of gp120 in the brain may increase the susceptibility to methamphetamine-induced neurotoxicity and exacerbate neuronal dysfunction in HIV+ individuals. For example, treating human neuronal cell cultures with a combination of gp120 protein and methamphetamine at physiologically inert doses when given alone led to significantly increased levels of neuronal cell death (Turchan et al, 2001). However, determining the specific role of gp120 in methamphetamineand HIV-induced cognitive impairments is extremely difficult, if not impossible, in humans because of the potential contributions of additional HIV-associated proteins such as TAT (Carey et al, 2012) and Nef (Chompre et al, 2013).

Transgenic mice with brain-specific expression of the gp120 protein are a useful tool for investigating the interactive effects of HIV and methamphetamine on cognitive function. Reactive astrocytosis, an important marker of brain injury (Eddleston and Mucke, 1993), has been observed in gp120-expressing mice (Toggas et al, 1994) and in postmortem HIV+ human brains (Vitkovic and daCunha, 1995). Cortical pyramidal neuron damage was found in both HIV+ humans (Achim et al, 2009) and gp120expressing mice (Toggas et al, 1994). Furthermore, gp120expressing mice show cognitive deficits, including impairments in contextual object recognition (Kesby et al, 2014b) and age-dependent spatial memory (D'Hooge et al, 1999; Hoefer et al, 2014). Limited studies have investigated the effects of methamphetamine in gp120-expressing mice. The expression of gp120 increases the sensitivity to methamphetamine-induced conditioned reward (Kesby et al, 2014a), alters stereotyped behavioral responses to acute methamphetamine (Roberts et al, 2010), and alters behavioral disinhibition after exposure to methamphetamine binge (Henry et al, 2013). Impairments in spatial strategy learning are exacerbated in gp120-expressing mice exposed to a methamphetamine binge (Hoefer et al, 2014; Kesby et al, 2014b). However, the combined effects of gp120 expression and methamphetamine binges on cognitive function have not been investigated using tests with cross-species relevance.

The goals of the present study were to (1) determine whether HIV-associated deficits in learning and executive functions are exacerbated in HIV+ humans with a history of methamphetamine dependence and (2) determine whether similar patterns of cognitive impairments are evident in gp120-expressing mice during long-term abstinence from methamphetamine/saline exposure. Learning and executive functions were assessed using a neurocognitive test battery in humans and the attentional-set-shifting task (ASST) in mice. Direct cross-species comparisons of learning and executive functions were made by utilizing analogous analyses to demonstrate the utility of such methods for future work in translational neuroscience.

\section{MATERIALS AND METHODS}

\section{Human Participants}

A total of 121 human participants, all adult men, were classified into four groups according to HIV seropositive $(\mathrm{HIV}+)$ or seronegative (HIV - ) status, and diagnostic criteria for lifetime methamphetamine dependence (METH+ or METH -). The groups consisted of $29 \mathrm{HIV}+/ \mathrm{METH}+, 25$ HIV - /METH+, 31 HIV+/METH - , and 36 HIV - /METH subjects. All of the METH+ subjects met the criteria for methamphetamine dependence according to the DSM-IV criteria measured by the Composite International Diagnostic Interview (CIDI) within 18 months of their study assessments. See Table 1 for group demographics, methamphetamine use characteristics, and psychiatric characteristics, Table 2 for HIV status and general medical characteristics, and Supplementary Methods for detailed inclusion criteria.

\section{Neurocognitive Assessments}

All of the participants completed a neurocognitive test battery that was constructed in line with the Frascati criteria for HIVassociated neurocognitive disorder (Antinori et al, 2007) and assessed the following seven domains: verbal fluency, executive functions, speed of information processing, learning, delayed recall, working memory, and motor skills (Heaton et al, 2010). Tests that contributed to learning domain scores included total learning measures from the Brief Visuospatial Memory Test-Revised and the Hopkins Verbal Learning TestRevised. Tests that contributed to executive function domain scores included the Halstead Category Test, Wisconsin Card Sorting Test (64 item), Trail Making Test (Part B) and Stroop Color-Word Interference Trial.

Raw neurocognitive test scores were converted to $T$-scores using demographically adjusted normative standards to account for the effects of age, education, and ethnicity (Norman et al, 2011). Demographically corrected $T$-scores were then converted into a global deficit score (GDS) and domain deficit scores (DDS) according to a standardized approach (Carey et al, 2004; Woods et al, 2008). As a separate measure of cognitive function relevant to the daily functioning of individuals that may not be reflected by cognitive performance scores, we assessed the rate of mild cognitive impairments. GDS and DDS $\geqslant 0.5$ were used to define global- and domain-specific neuropsychological impairments, respectively.

\section{Mice}

Fifty-one male mice (4-5 months old at the start of the methamphetamine regimen) that expressed the gp120 protein (Toggas et al, 1994) under the regulatory control of a modified murine glial fibrillary acidic protein (25 gp120 mice and 26 gp120+ mice) and previously tested in a battery of cognitive tasks (Kesby et al, 2014b), excluding the ASST, were evaluated (see Supplementary Methods). All of the experiments were conducted in accordance with the guidelines of the American Association for the Accreditation of Laboratory Animal Care and National Research Council's Guide for the Care and Use of Laboratory Animals and approved by the University of California San Diego Institutional Animal Care and Use Committee. 
Table I Demographic and Psychiatric Characteristics of the Human Study Sample

\begin{tabular}{|c|c|c|c|c|c|c|}
\hline & $\begin{array}{c}\text { HIV }-I \\
\text { METH }-(n=36)\end{array}$ & $\begin{array}{l}\text { HIV - /METH+ } \\
\quad(n=25)\end{array}$ & $\begin{array}{c}\text { HIV+l } \\
\text { METH }-(n=3 I)\end{array}$ & $\begin{array}{l}\text { HIV+/METH+ } \\
\quad(n=29)\end{array}$ & $\mathbf{F} / \chi^{2}$ & $\mathbf{P}$ \\
\hline \multicolumn{7}{|l|}{ Demographic characteristics } \\
\hline Education (years) & I3.7 (2.1) & $12.0(1.7)^{*}$ & $14.0(2.0)$ & | $3.3(2.4)$ & 4.52 & 0.005 \\
\hline WRAT reading score & $103.2(11.9)$ & 99.1 ( 10.47$)$ & $101.4(10.1)$ & I04.8 ( 1 1.6) & 1.33 & 0.268 \\
\hline \multicolumn{7}{|c|}{ Methamphetamine use characteristics } \\
\hline Age of first use (years) ${ }^{\mathrm{a}}$ & - & $23.0(8.4)$ & - & $24.1(7.3)$ & 0.28 & 0.596 \\
\hline Duration of use (years) ${ }^{a}$ & - & $6.6(5.8)$ & - & $6.2(6.9)$ & 0.04 & 0.843 \\
\hline Last use (years) ${ }^{a}$ & - & $0.3(0.4)$ & - & $0.4(0.4)$ & 0.62 & 0.434 \\
\hline BDI-II & $2.7(3.8) *$ & $19.2(12.4)^{\#}$ & $11.0(\mid 1.2)$ & |3.9(|3.6) & 13.14 & $<0.00$ । \\
\hline BAl total score & $22.2(1.9)^{*}$ & $29.8(8.2)$ & $27.7(7.9)$ & $28.9(9.0)$ & 7.52 & $<0.001$ \\
\hline Substance dependence $e^{b, c}$ & $8.3 \%$ & $60.0 \% \%^{\#, \wedge}$ & $16.1 \%$ & $34.5 \% \wedge$ & 22.74 & $<0.001$ \\
\hline POMS total & $32.4(29.7)^{*}$ & $85.0(40.7)^{*}$ & $55.7(37.8)$ & $68.5(51.6)$ & 9.35 & $<0.001$ \\
\hline
\end{tabular}

Abbreviations: BAI, Beck Anxiety Inventory; BDI-II, Beck Depression Inventory-II; METH, methamphetamine; POMS, Profile of Mood States; WRAT, Wide Range

Achievement Test, 3rd Edition-reading portion.

${ }^{*} P<0.05$, compared with all other groups; ${ }^{\#} P<0.05$, compared with $H I V+/ M E T H-$; ${ }^{\wedge} P<0.05$, compared with HIV $-/ M E T H$.

The data are expressed as mean (SD) unless otherwise indicated.

${ }^{a}$ Statistical comparisons were made between METH+ groups (see Supplementary Methods).

benotes lifetime diagnosis

'Excluding methamphetamine dependence

Table 2 HIV Disease and Medical Characteristics of the Human Study Sample

\begin{tabular}{|c|c|c|c|c|c|}
\hline & HIV+/METH $-(n=3 I)$ & HIV+/METH+ $(n=29)$ & $\mathbf{F} / \chi^{2}$ & $P$ & \\
\hline \multicolumn{6}{|l|}{ HIV disease characteristics } \\
\hline AIDS (\%) & $35.5 \%$ & $55.2 \%$ & 2.36 & 0.124 & - \\
\hline Current ART use (\%) & $56.7 \%$ & $60.7 \%$ & 0.10 & 0.754 & - \\
\hline ARV regimen duration (months) & $26.2(26.7)$ & $21.6(18.3)$ & 0.33 & 0.568 & - \\
\hline HIV duration (months) & $84.8(98.8)$ & $123.8(\mid 04.7)$ & 0.11 & 0.738 & - \\
\hline Plasma HIV RNA $>50 \mathrm{c} / \mathrm{ml}$ & $44.8 \%$ & $51.7 \%$ & 0.28 & 0.599 & - \\
\hline CSF HIV RNA $>50 \mathrm{c} / \mathrm{ml}$ & $39.1 \%$ & $47.4 \%$ & 0.29 & 0.591 & - \\
\hline
\end{tabular}

Abbreviations: AIDS, acquired immunodeficiency syndrome; ART, antiretroviral therapy; CSF, cerebrospinal fluid; METH, methamphetamine; RNA, ribonucleic acid.

The data are expressed as mean (SD) unless otherwise indicated.

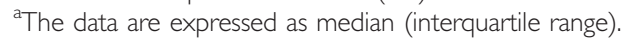

Methamphetamine regimen. Methamphetamine hydrochloride (Sigma, St Louis, MO) was dissolved in saline $(0.9 \%)$ and administered subcutaneously in a $5 \mathrm{ml} / \mathrm{kg}$ injection volume. The mice were administered an escalating dose-multiple binge methamphetamine regimen $(\mathrm{METH}+)$ or saline (METH -; see Supplementary Methods; (Kesby et al, 2014b)). The mice were tested in the ASST 2 months after completing the methamphetamine regimen, which is equivalent in time to several years for an adult human
(Andreollo et al, 2012). Thus, the focus of the study in both mice and human subjects was the persistent effects of previous methamphetamine exposure rather than the effects of acute methamphetamine or early abstinence.

Attentional-set-shifting task. Learning and executive functions were assessed in mice using the ASST (Young et al, 2010) based on the human intradimensional/ 
extradimensional shift task (Birrell and Brown, 2000; see Supplementary Methods). Testing was conducted 2 months after the completion of the methamphetamine regimen and when the mice were 7-8 months of age. Mice were food restricted to $85 \%$ free-feeding weight 10 days before and during testing in the ASST. The mice were rewarded with regular food pellets rather than sugar pellets (Young et al, 2010). Briefly, specific odors and platform textures were used as cues to guide discrimination learning. The stages of the task included the following (in order): simple discrimination, compound discrimination, compound reversal, intradimensional shift, intradimensional reversal, and extradimensional shift (Supplementary Table S1). At each stage, the mice were required to make six consecutive correct responses before moving to the next stage. A mouse failed the task if six consecutive correct trials were not achieved after 80 trials in a discrimination stage or 100 trials in a reversal stage. The mice were counterbalanced so that the initial discrimination would be either odor or platform texture. Stimulus combinations and locations were selected in a balanced order. The number of trials to reach criterion was recorded for each stage.

\section{Statistical Analyses}

Human studies. Pearson's $\chi^{2}$-analyses were used to compare the impairment rates of human subjects (with 95\% confidence interval (CI) estimates) in each cognitive domain. Learning and executive function DDS were analyzed using a two-way analysis of variance (ANOVA), with HIV and Methamphetamine as the between-subjects factors. Tscores for each domain-specific test were analyzed using repeated measures (RM)-ANOVAs, with HIV and Methamphetamine as the between-subjects factors and Test as the RM. Where significant effects were detected, psychiatric characteristics were also tested for covariate effects. Demographic and disease-related variables were examined using between-group $\chi^{2}$-tests for binary variable or ANOVAs for continuous variables.

Mouse studies. Survival curves (ie, the number of subjects successfully completing each stage) were estimated using the Kaplan-Meier technique and compared using the log-rank
(Mantel-Cox) test. Pearson's $\chi^{2}$-analyses were used to compare the overall failure rate of mice in the ASST. Learning (consisting of three discrimination learning stages: simple, compound, and intradimensional shift), executive functions (consisting of the two reversal learning stages: compound and intradimensional) and $T$-scores for each ASST stage were analyzed using RM-ANOVAs, with dimension, genotype, and methamphetamine as the between-subjects factors and stage as the RM.

General statistics. When appropriate, post hoc comparisons of means were performed using Least Significant Difference analyses. All of the results are expressed as mean \pm SEM unless otherwise indicated. Differences were considered statistically significant at $P<0.05$. All of the analyses were performed using SPSS Statistics v.19 software (Chicago, IL).

\section{RESULTS}

\section{Human Impairment Rates}

Within the domain of learning, a significant difference was found between groups $\left(\chi_{3}^{2}=8.8, P<0.05\right.$; Figure $\left.1 b\right)$. A step-wise increase in impairment between the groups was observed, with the HIV-/METH - group showing the lowest impairment (28\%; CI, 13-42\%) followed by the HIV-/METH+ group (35\%; CI, 16-53\%) and the HIV+/METH - group (44\%; CI, 26-61\%), with the greatest level of impairment in the HIV+/METH+ group $(66 \%$; CI, 48-83\%). Post hoc tests confirmed that the HIV+/METH+ group was more likely to be impaired than either the HIV-I METH - $(P<0.01)$ or the HIV-/METH+ $(P<0.05)$ group. Similar stepped profiles of impairment were observed for GDS (Figure 1a) and executive function's DDS (Figure 1c), but the differences in impairment rates between groups were not statistically significant.

\section{Mouse Failure Rates}

The percentage of subjects that failed to successfully reach criterion during any stage of the task is a commonly used metric of cognitive impairment (Leeson et al, 2009). Thus,

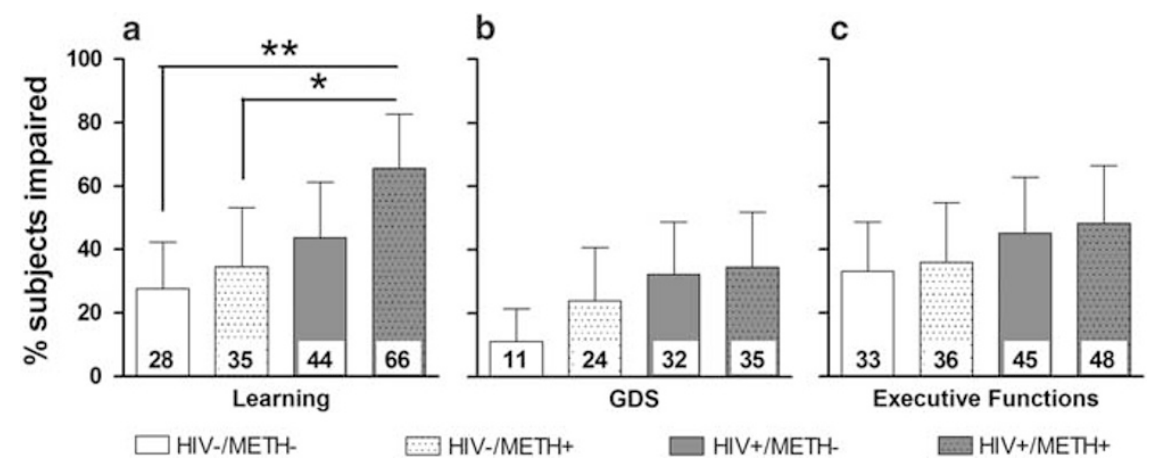

Figure I Levels of cognitive impairment in humans ( $n=25-36$ per group). Effects of HIV and methamphetamine (METH) dependence on the percentage of human subjects considered impaired as reflected by their learning deficit scores (a), global deficit scores (GDS) (b) and executive functions deficit scores (c). A significantly greater proportion of the dual $\mathrm{HIV}+/ \mathrm{METH}+$ subjects were classified as impaired on learning tasks compared with both $\mathrm{HIV}-$ groups. Although a similar pattern was evident in both GDS and executive functions deficit scores, no significant group differences were observed. Each data point represents the percentage of the group total with $95 \%$ confidence intervals $\left(\chi^{2}\right.$-analyses, $* P<0.05, * * P<0.0 I$ ). 
we analyzed the percentage of mice that failed to complete the ASST (ie, were unable to make six consecutive correct choices within the trial limit during any stage) as a categorical measure, reflecting greater learning impairment compared with mice that completed the task. The number of failures differed depending on dimension, genotype, and methamphetamine exposure (Figure 2), precluding analyses of one aspect of executive functions (attentional set shifting). Therefore, in mice that successfully completed the ASST, the analyses focused on the discrimination stages as measures of learning and the reversal stages as measures of executive functions.

Platform texture discrimination was more difficult than odor discrimination for all of the mice. Significantly more mice failed to complete all of the test stages when the original dimension was platform texture (22\%) compared with odor $\left(54 \% ; \chi^{2}{ }_{1}=6.5, P<0.05\right)$. Nearly half $(45 \%)$ of all of the failures occurred in the initial simple discrimination stage, suggesting that the failure rates can be attributed to learning impairments rather than executive functions impairments. A significant difference was observed between experimental groups in the failure rate across the ASST stages $\left(\chi_{7}^{2}=15.3\right.$, $P<0.05$; Figure $2 \mathrm{a}$ ), with the greatest failure rate observed in the gp120+/METH+ (Figure 2b).

\section{Learning in Humans}

HIV+ subjects exhibited significantly worse learning than HIV - subjects, as reflected by a higher average learning DDS (main effect of HIV: $\mathrm{F}_{1,117}=7.4, P<0.01, d=0.50$; Figure $3 \mathrm{a}$ ) and lower average $T$-scores (main effect of HIV: $\mathrm{F}_{1,112}=4.3, P<0.05, d=0.48$; Figure $3 \mathrm{~b}$ ). Methamphetamine dependence and time since last methamphetamine use (see Table 1) were not associated with any differences in learning performance in this cohort, regardless of HIV status. Furthermore, this impairment was not associated with any differences in psychiatric characteristics, such as major depressive disorder or mood/anxiety scores (see Table 1). For individual test results, see Figure 4a,b.

\section{Learning in Mice}

During the discrimination (learning) stages of the ASST, the gp120+ mice required more trials to reach criterion than gp120 - mice (main effect of Genotype: $\mathrm{F}_{1,25}=4.7, P<0.05$; Figure 3e) indicating deficits in learning. Similar to our human findings, methamphetamine exposure did not worsen learning performance. Consistent with increased failure rates in platform texture discrimination, a significant main effect of Dimension $\left(\mathrm{F}_{1,25}=34.0, P<0.001\right)$ and $\mathrm{a}$ significant Stage $\times$ Dimension interaction $\left(\mathrm{F}_{2,50}=4.8\right.$, $P<0.05)$ were detected. The initial stages required more trials to discriminate platform texture compared with odor (Figure 5a). No significant effects of dimension, methamphetamine, or genotype on the latencies to respond were observed (Figure 5b).

To account for the differences in difficulty across dimensions and stages, the total trials to reach criterion in each stage were converted to $T$-scores using overall means and SDs for the relevant dimension (ie, odor or platform texture). The analyses revealed that gp120+ mice had lower average $T$-scores than gp120- mice (main effect of Genotype: $F_{1,29}=4.5, P<0.05$; Figure $3 f$ ). No significant effects of methamphetamine were observed.

\section{Executive Functions in Humans}

In contrast to the learning domain, neither HIV nor Methamphetamine was significantly associated with executive functioning, based either by DDS (Figure 3c) or T-scores (Figure 3d). However, small effect sizes were associated with HIV for both executive functions DDS $(d=0.22)$ and executive functions $T$-scores $(d=0.21)$ suggesting that impairments may have been detectable in a larger cohort of subjects (see also Figure 1c). For individual test results, see Figure $4 c-f$.
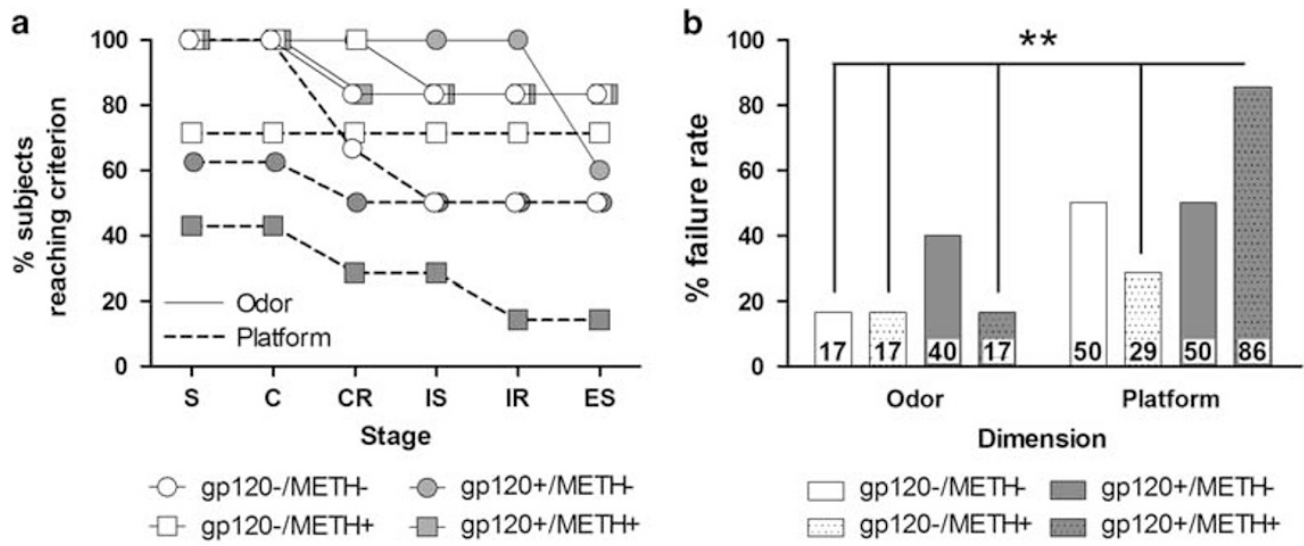

Figure 2 Levels of learning impairment in mice separated based on gp I 20 expression, methamphetamine $(M E T H)$ exposure, and dimension $(n=5-8$ per group). (a) Survival curves for mice across each stage of the attentional-set-shifting task. A greater rate of failure was observed for mice in platform texture discrimination compared with the odor discrimination stages, suggesting that platform texture discrimination was more difficult than odor discrimination. (b) The overall failure rate was greatest in the dual gp I20+/METH+ group that was discriminating between platform textures compared with the majority of the other groups, particularly those performing odor discrimination. Each data point represents the percentage of the group total $\left(\chi^{2}\right.$ analyses, $* * *<0.0$ I compared with the platform texture discriminating gp/20+/METH+ group). C, compound; CR, compound reversal; ES, extradimensional shift; IS, intradimensional shift; IR, intradimensional reversal; S, simple. 


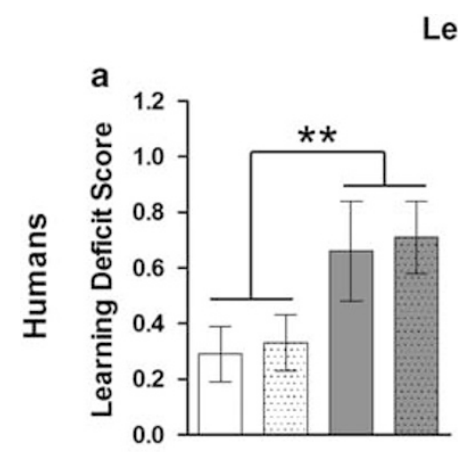

\section{Learning}

b
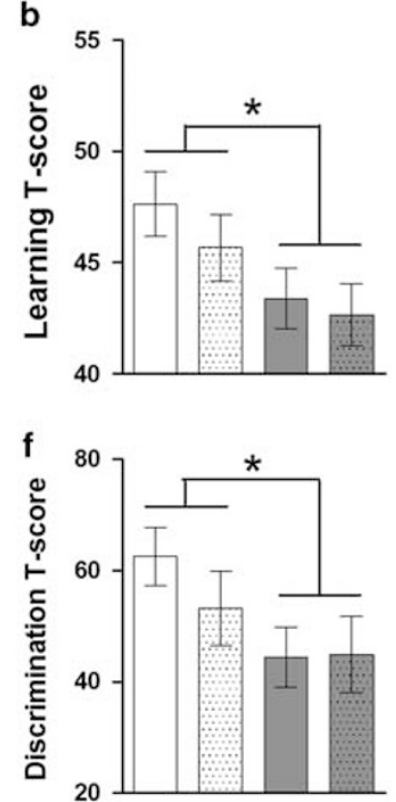

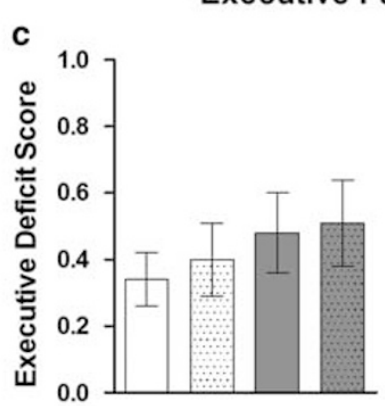

d

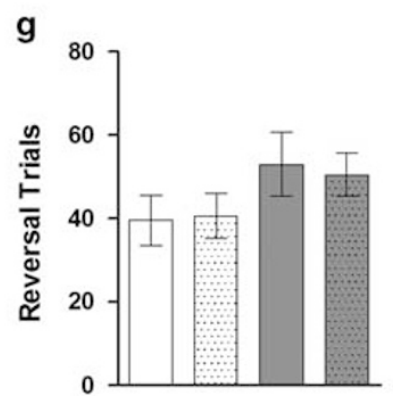

h
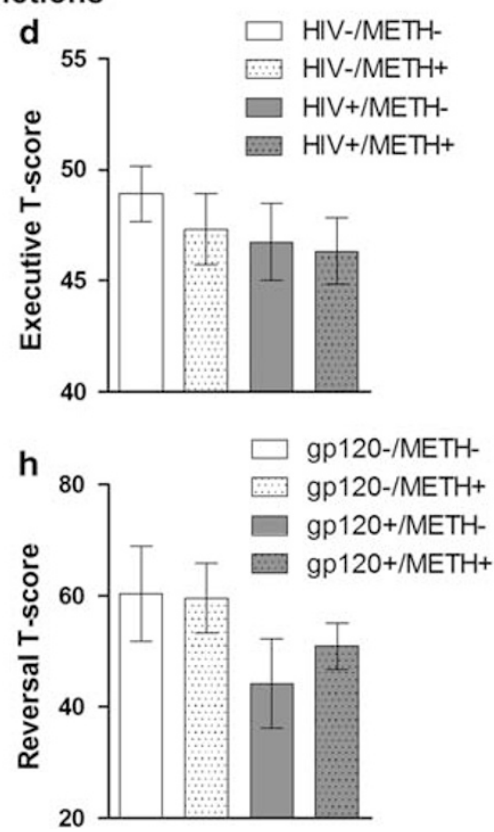

Figure 3 Learning ( $a, b$, e, and $f$ ) and executive functions ( $c, d$, g, and $h$ ) in humans (top panels; $n=25-36$ per group) and mice (bottom panels; $n=6-10$ per group). (Top left) Effects of HIV and methamphetamine dependence on domain deficit scores (DDS) (a) and demographically adjusted T-scores (b) for learning tasks in human subjects. The learning DDS were significantly higher in HIV+ subjects, and T-scores were significantly lower in HIV+ subjects, regardless of methamphetamine dependence. (Top right) Effects of HIV and methamphetamine (METH) dependence on executive functions in human subjects ( $c$ and d). No significant effects of HIV or methamphetamine dependence were found on either DDS (c) or adjusted T-scores (d) for executive functions in human subjects. (Bottom left) Effects of gpl20 expression and methamphetamine exposure on discrimination learning (e) and adjusted T-scores for discrimination learning $(f)$ in mice. The expression of gp I 20 in mice led to significantly more trials required to reach criterion in a discrimination learning task, regardless of methamphetamine exposure. Dimension-adjusted T-scores for discrimination learning were significantly lower in gp I 20+ mice regardless of methamphetamine exposure. (Bottom right) Effects of gp I 20 expression and methamphetamine exposure on reversal learning in mice (g and $\mathrm{h}$ ). Similar to the findings in humans, no significant effects of gp 120 expression or methamphetamine exposure were found on trials to reach criterion (g) or adjusted $T$-scores (h) for reversal learning in mice. The data are expressed as mean \pm SEM. $* P<0.05, * * P<0.0$ I

\section{Reversal Learning (Executive Functions Component) in Mice}

No significant effects of Dimension, Stage, Genotype, or Methamphetamine on either the number of trials to reach criterion (Figure 3g) or adjusted $T$-scores (Figure $3 \mathrm{~h}$ ) during the reversal learning stages were detected.

\section{DISCUSSION}

The present cross-species study revealed similar learning and executive functions impairment patterns in human subjects with HIV disease and/or methamphetamine dependence and mice with gp120 protein expression and/or methamphetamine exposure. Specifically, learning deficits were observed in both HIV+ humans and gp120+ mice, whereas executive functions were not significantly affected in either group. Furthermore, no persistent effect of methamphetamine exposure on learning or executive functions was observed in either species. Interestingly, however, the greatest rate of learning impairment was observed in HIV+/METH+ human subjects. Similarly, the greatest failure rate in the ASST was observed in gp120-expressing and methamphetamineexposed mice when tested in a complex platform texturebased discrimination task.

\section{HIV Disease and Methamphetamine Dependence in Humans}

The present work demonstrated that HIV disease was associated with prominent deficits in the domain of learning but not in executive functions. These findings are consistent with previous observations of learning impairments associated with HIV disease without concomitant impairments in executive functions (Rippeth et al, 2004). However, deficits in executive functions have been frequently reported in HIV+ subjects (Heaton et al, 1995, 2011; Marquine et al, 2014). Sample characteristics in the current cohort, including modest global impairment rates in the risk groups (24-35\%; Figure 1a) may preclude the detection of small effect sizes (such as those observed in the present study) and contribute to these discrepancies. The HIV+ groups in previous studies (Heaton et al, 1995, 2011; Rippeth et al, 2004) tended to be much larger and had more advanced HIV and greater overall levels of impairments, than the cohort used in the present study. Furthermore, executive functions represent a collection of cognitive abilities that are involved in the maintenance and control of cognitive and behavioral function and considered to be dependent on the prefrontal cortex (Anderson and Tranel, 2002). For example, HIV-associated impairment in one (Trail Taking Test, Part B; see Figure 4c) of the four executive functions tests in the present study 

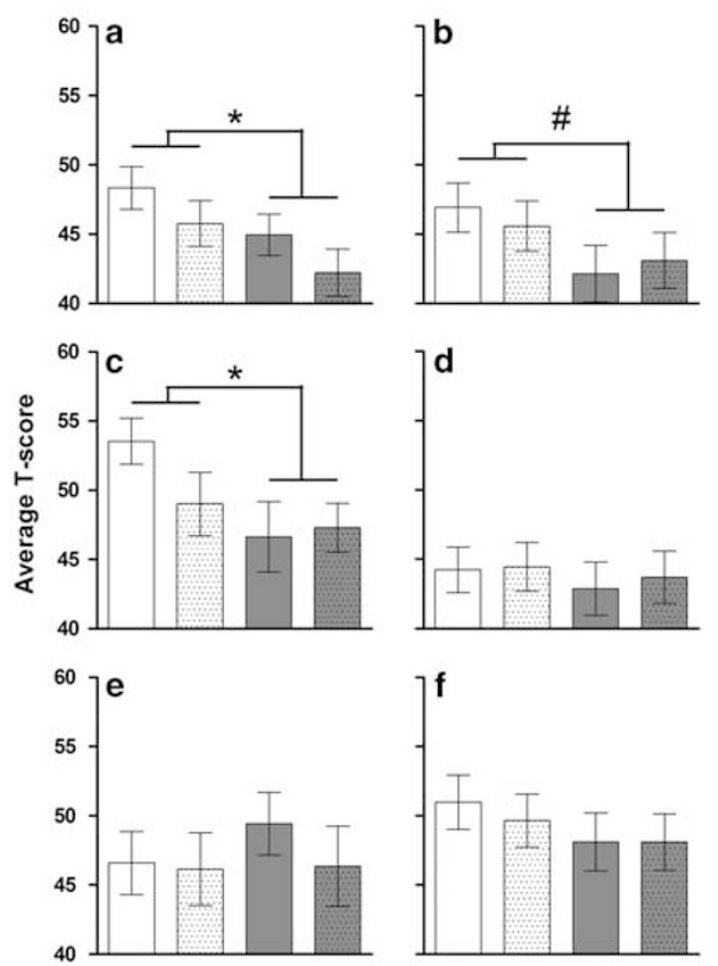

$\square$ HIV-/METH $\square$ HIV-/METH+ $\square$ HIV+/METH- 0 HIV+/METH+

Figure 4 T-scores for the individual neurocognitive tests $(n=25-36$ per group). Tests that contributed to learning domain scores included the Brief Visuospatial Memory Test-Revised, trials I-3 (a), and the Hopkins Verbal Learning Test-Revised, trials I-3 (b). Tests that contributed to executive functions domain scores included the Trail Making Test (Part B; c), Halstead Category Test (d), Wisconsin Card Sorting Test (64 item; e), and Stroop Color-Word Interference Trial (f). HIV was associated with significantly decreased performance on the Brief Visuospatial Memory Test ( $a ; F_{1,117}=$ 4.7, $P<0.05, d=0.42)$ and the Trail Making Test $\left(c ; F_{1,116}=4.3, P<0.05\right.$, $d=0.42$ ). A trend toward a similar HIV-associated decrease in performance was also observed in the Hopkins Verbal Learning Test (b; $F_{1,116}=3.5$, $P<0.1, d=0.36)$. The data are expressed as mean \pm SEM. $* P<0.05$, ${ }^{\#} P<0.1$

suggests that this aspect of executive functioning may be more susceptible to HIV-induced impairments. Overall, however, consistent with other studies (Heaton et al, 1995, 2011), the present results suggest that deficits in executive functions in HIV+ subjects are less ubiquitous than deficits in learning. The low level of global impairment levels in the current cohort further suggest that deficits in learning are present at earlier disease stages than deficits in executive functions and thus, may help identify subjects at risk for further cognitive decline.

Detecting the deleterious effects of methamphetamine dependence on cognitive function in uninfected subjects remains challenging (Hart et al, 2012). As a consequence, the interactive effects of HIV disease and methamphetamine dependence on cognitive function are also difficult to demonstrate. In the present study, methamphetamine dependence had no effect on cognitive performance, regardless of HIV status. The combination of methamphetamine dependence and HIV disease, however, resulted in the greatest rate of impairment in learning among patient groups. Importantly, these data would suggest that the
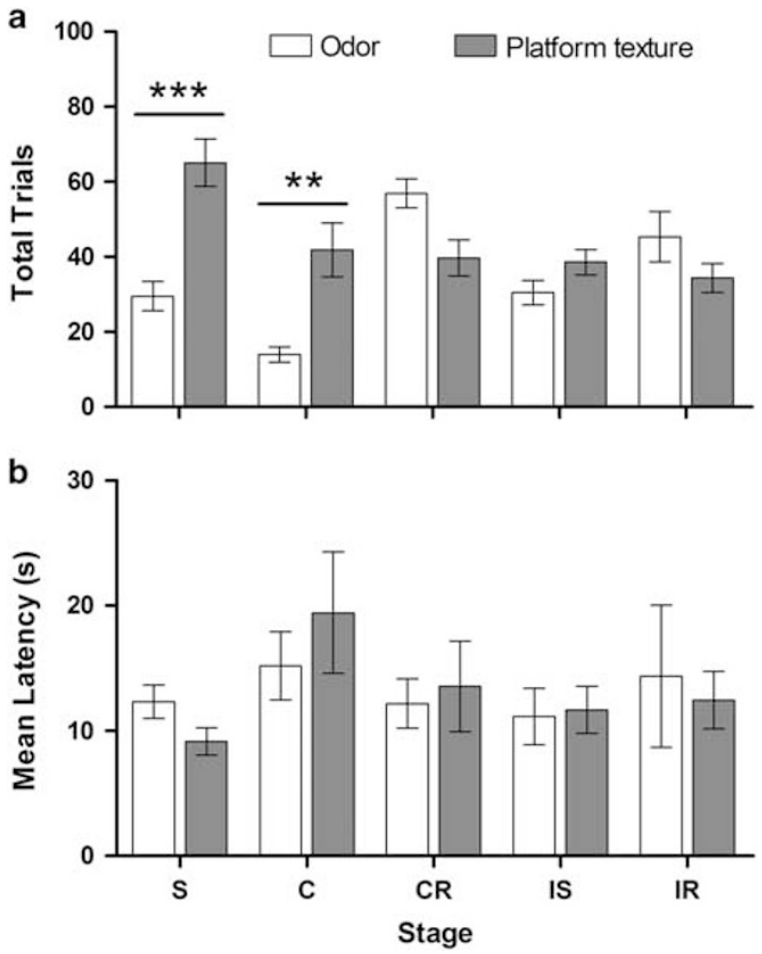

Figure 5 Effects of dimension on trials to criterion and latency to respond (odor: $n=20$; platform texture: $n=13$ ). (a) More total trials were required to reach criterion when successfully discriminating between platform textures compared with odors in the discrimination stages of the ASST, specifically the simple and compound discrimination stages. (b) Mice showed similar latencies to respond regardless of dimension. The data are expressed as mean $\pm \mathrm{SEM}$. $* * P<0.0$ I, ***P $<0.00$ I. ASST, attentional-setshifting task; C, compound; CR, compound reversal; IS, intradimensional shift; $\mathbb{R}$, intradimensional reversal; $S$, simple.

combination of HIV disease and methamphetamine dependence increases the susceptibility to mild cognitive impairments but not the severity of these impairments per se. Performance scores for learning were not significantly affected by methamphetamine dependence (see Figure 3), even though performance in the Brief Visuospatial Memory Test-Revised (see Figure 4a) did show a similar pattern to that observed in the impairment rates. Although a similar trend was observed in the impairment rates for executive functions, this pattern of results suggests that the domain of learning may be more sensitive to both HIV and the combination of HIV and methamphetamine than the domain of executive functions. A previous meta-analysis of human studies found that the domain of learning is the most sensitive to methamphetamine-induced cognitive impairments (Scott et al, 2007). Consistent with these findings, we observed subtle effects in the domain of learning, but not on executive functions. However, executive functions may be significantly affected by the combination of HIV and/or methamphetamine dependence in subgroups of people (Bousman et al, 2010; Gupta et al, 2011). As a result, the putative interaction between HIV and methamphetamine dependence may be obscured by sampling artifact. 


\section{gp120 Expression and Methamphetamine Exposure in Mice}

Brain-specific expression of the HIV-associated gp120 protein led to impaired discrimination learning in mice in the ASST. Studies that investigated the effects of gp 120 expression on cognitive function are sparse. gp120expressing mice have exhibited impairments in spatial learning/memory in the Morris water maze (D'Hooge et al, 1999) and contextual object recognition in the object-inplace task (Kesby et al, 2014b). Importantly, in the latter study, assessments of anxiety-like behavior and, novel object and location recognition memory were not impaired by gp120 expression (Kesby et al, 2014b). Thus, impaired discrimination learning in gp120-expressing mice can be attributed to impaired associative learning and not due to anxiogenic behavior, or an inability to differentiate olfactory (odors) or tactile (platform textures) stimuli. A key finding of the present study was that the rate of gp120-induced learning impairments were exacerbated by prior methamphetamine exposure, but this was demonstrated only under experimental conditions with increased task difficulty (ie, platform texture $v s$ odor discrimination). Similarly, in our previous work, exacerbated cognitive deficits in spatial strategy learning induced by combined exposure to gp120 and methamphetamine were observed during the later but not early stages of task acquisition in the Barnes maze (Kesby et al, 2014b). Thus, specific testing conditions or tasks with higher cognitive demands may be required to reveal the combined effects of gp120 and methamphetamine on cognitive function.

The mechanisms that may underlie the observed deficits in learning associated with gp120 expression and the increased susceptibility to learning impairments by gp120 expression and methamphetamine exposure are currently unknown. Multiple brain regions and associated circuitry contribute to associative learning performance including, but not limited to, the prefrontal cortex (Leon et al, 2010), hippocampus (Kim et al, 2011), and striatum (Steinberg et al, 2013). However, dopamine neurons have been found to be critically involved in multiple aspects of associative or cue-reward learning (Steinberg et al, 2013), suggesting that altered dopaminergic function caused by both HIV/gp120 and methamphetamine exposure may impair learning performance. Both gp120 and methamphetamine are known to impact dopamine function, specifically at the dopamine transporter. For example, methamphetamine dependence in humans (Panenka et al, 2013) or exposure to methamphetamine binges in rats (Segal and Kuczenski, 1997) leads to decreases in subcortical dopamine transporter levels and dopamine levels, respectively, that persist after prolonged periods of abstinence. Exposing human mesencephalic neuronal/glial cultures to gp120 results in both decreased dopamine uptake and increased cell death (Hu et al, 2009). gp120-induced effects in the latter study were specific to dopaminergic neurons, highlighting their preferential sensitivity to gp120 exposure. Previous observations of increased sensitivity to methamphetamine-conditioned reward in gp120-expressing mice (Kesby et al, 2014a) provide additional evidence that the dopaminergic response to methamphetamine may be altered by gp120 expression. However, other neurotransmitter systems, such as glutamate and $\gamma$-aminobutyric acid, have also been associated with gp120induced neurotoxicity (Fernandes et al, 2007; Hoefer et al, 2014) and may also contribute to impaired cognitive function.

Unlike in previous studies on C57BL/6N mice (Young et al, 2010), a high failure rate was observed in the ASST, especially for the platform texture dimension. Therefore, the ability to perform an attentional-set-shift was not evaluated, precluding a more direct comparison with the Wisconsin Card Sorting Test in humans. Reversal learning was similar in gp120expressing and/or methamphetamine-exposed mice suggesting that this aspect of their executive functions was not impaired. In our previous work, subtle effects of gp120 expression and/or methamphetamine exposure, but no interactive effects, were observed in spatial reversal learning in the Barnes maze task (Kesby et al, 2014b). Because executive functions represent a group of cognitive abilities (Anderson and Tranel, 2002), the lack of gp120- or methamphetamine-induced deficits in reversal learning in the ASST does not preclude specific deficits in other prefrontal cortex-based cognitive tasks. Further testing of broader aspects of executive functions in these mice is warranted.

\section{Comparative Analyses of Data from Humans and Mice}

All rodent models of HIV disease have limitations (Jaeger and Nath, 2012) that complicate direct comparisons with HIV disease in human subjects. Our comparative analyses of learning and executive functions in humans and mice, provided evidence that the gp120-expressing mouse, while not reproducing all aspects of the disease, provides a viable translational tool for investigating the persistent mild cognitive deficits associated with HIV disease in the presence of combination antiretroviral therapy (Heaton et al, 2011), particularly when gp120 levels in serum and tissue remain detectable even in infected subjects with low or undetectable levels of viral replication (Santosuosso et al, 2009).

A major focus of both clinical and basic research on disease-related cognitive outcomes is the development of translational tests that can be directly compared between species. A growing number of translational tests have been developed in both rodents and humans including the ASST (Leeson et al, 2009; Young et al, 2010), the Behavioral Pattern Monitor (Young et al, 2007), the Response Bias Probabilistic Reward Task (Der-Avakian et al, 2013), and the 5-choice Continuous Performance Test (Young et al, 2013) among others. Clinical data sets face confounds such as variations in age and the use of other substances that can be controlled in animal studies. To account for demographic factors, human data are commonly converted into demographically corrected $T$-scores (Heaton et al, 2004). To address the discrepancies in behavioral measures in humans and animals, behavioral measures can be similarly combined into domain-specific scores. Applying this methodology to the ASST data in mice allowed us to normalize the difference in difficulty between odor and platform texture discrimination. Considering that different tests of learning were used to generate $T$-scores in each species, after this correction, the effects of gp120 expression on learning $T$-scores were remarkably similar to those found in HIV+ humans. Thus, this methodology has the potential to be used with behavioral test batteries in animal models to produce domain-specific outcomes, allowing for analyses that are identical to human 
data sets. Regardless of the translational value of a specific task, this approach may provide a more sensitive method for the validation of animal models of disease.

\section{CONCLUSION}

In summary, this is the first cross-species study that has investigated the combined effects of HIV/gp120 and methamphetamine on cognition. Similar findings in $\mathrm{HIV}+$ humans and gp120-expressing mice suggest that gp120 protein expression may contribute to the observed learning deficits in HIV+ humans. Furthermore, combined methamphetamine exposure- and gp120-induced neurotoxicity may increase vulnerability to mild cognitive impairment. The use of cross-species analyses of behavioral data sets between humans and relevant animal models, such as gp120-expressing mice, may help to detect cognitive domains that are particularly sensitive to injury induced by HIV, methamphetamine, or their combination. Moreover, the potential neurobiological mechanisms that underlie these deficits may be identified in future work, thus assisting in the development of adjunct therapeutics to treat mild cognitive impairments (especially those that involve learning) in methamphetamine-dependent and HIV+ humans.

\section{FUNDING AND DISCLOSURE}

This work was supported by the Translational Methamphetamine AIDS Research Center funded by the National Institute on Drug Abuse (TMARC P50 DA26306) and the Interdisciplinary Research Fellowship in NeuroAIDS (IRFN, MH81482 to JPK). JWY has received funding support from Lundbeck and Omeros and consultancy support from Amgen. AM has received contract research support from Forest Laboratories and Astra-Zeneca and honoraria/ consulting fees from AbbVie during the past 2 years. AM and SS have a patent on the use of metabotropic glutamate compounds for the treatment of nicotine dependence that is unrelated to the present research. SLL has received research support from Abbvie, Gilead Sciences, and ViiV Healthcare in the past 36 months. SPW has received support from Merck, Posit Science, and Oxford University Press. IG has received compensations from Abbot Laboratories, Abbvie and Merck. All authors declare no conflict of interest.

\section{ACKNOWLEDGMENTS}

We thank Mr Michael Arends for outstanding editorial assistance. The Translational Methamphetamine AIDS Research Center (TMARC) is affiliated with the University of California, San Diego (UCSD), and the Sanford-Burnham Medical Research Institute (SBMRI). The TMARC is comprised of: Director-Igor Grant; Co-Directors-Ronald J EllisScott L Letendre, and Cristian L Achim; Center Manager-Steven Paul Woods; Assistant Center ManagerAaron M Carr; Clinical Assessment and Laboratory (CAL) Core: Scott L Letendre (Core Director), Ronald J Ellis, Rachel Schrier; Neuropsychiatric (NP) Core: Robert K Heaton (Core Director), J Hampton Atkinson, Mariana Cherner, Thomas D Marcotte, Erin E Morgan; Neuroimaging (NI)
Core: Gregory Brown (Core Director), Terry Jernigan, Anders Dale, Thomas Liu, Miriam Scadeng, Christine Fennema-Notestine, Sarah L Archibald; Neurosciences and Animal Models (NAM) Core: Cristian L Achim (Core Director), Eliezer Masliah, Stuart Lipton, Virawudh Soontornniyomkij; Administrative Coordinating Core (ACC)Data Management and Information Systems (DMIS) Unit: Anthony C Gamst (Unit Chief), Clint Cushman (Unit Manager); ACC-Statistics Unit: Ian Abramson (Unit Chief), Florin Vaida, Reena Deutsch, Anya Umlauf; ACC-Participant Unit: J Hampton Atkinson (Unit Chief), Jennifer Marquie-Beck (Unit Manager); Project 1: Arpi Minassian (Project Director), William Perry, Mark Geyer, Brook Henry; Project 2: Amanda B Grethe (Project Director), Martin Paulus, Ronald J Ellis; Project 3: Sheldon Morris (Project Director), David M Smith, Igor Grant; Project 4: Svetlana Semenova (Project Director), Athina Markou, James P Kesby; Project 5: Marcus Kaul (Project Director). The views expressed in this article are those of the authors and do not reflect the official policy or position of the United States government.

\section{REFERENCES}

Achim CL, Adame A, Dumaop W, Everall IP, Masliah E (2009). Increased accumulation of intraneuronal amyloid b in HIVinfected patients. J Neuroimmune Pharm 4: 190-199.

Anderson SW, Tranel D (2002). Neuropsychological consequences of dysfunction in the human dorsolateral prefrontal cortex. In: Grafman J (ed). The Frontal Lobes, 2nd edn. Elsevier: Amsterdam. Vol 7, pp 145.

Andreollo NA, Santos EF, Araujo MR, Lopes LR (2012). Rat's age versus human's age: what is the relationship? Arq Bras Cir Dig 25: $49-51$.

Antinori A, Arendt G, Becker JT, Brew BJ, Byrd DA, Cherner M et al (2007). Updated research nosology for HIV-associated neurocognitive disorders. Neurology 69: 1789-1799.

Aylward EH, Henderer JD, McArthur JC, Brettschneider PD, Harris GJ, Barta PE et al (1993). Reduced basal ganglia volume in HIV-1 associated dementia: results from quantitative neuroimaging. Neurology 43: 2099-2104.

Birrell JM, Brown VJ (2000). Medial frontal cortex mediates perceptual attentional set shifting in the rat. J Neurosci 20: 4320-4324.

Bousman CA, Cherner M, Glatt SJ, Atkinson JH, Grant I, Tsuang MT et al (2010). Impact of COMT Val158Met on executive functioning in the context of HIV and methamphetamine. Neurobehav HIV Med 2010: 1-11.

Carey AN, Sypek EI, Singh HD, Kaufman MJ, McLaughlin JP (2012). Expression of HIV-Tat protein is associated with learning and memory deficits in the mouse. Behav Brain Res 229: 48-56.

Carey CL, Woods SP, Gonzalez R, Conover E, Marcotte TD, Grant I et al (2004). Predictive validity of global deficit scores in detecting neuropsychological impairment in HIV infection. J Clin Exp Neuropsychol 26: 307-319.

Carrico AW (2011). Substance use and HIV disease progression in the HAART era: implications for the primary prevention of HIV. Life Sci 88: 940-947.

Chana G, Everall IP, Crews L, Langford D, Adame A, Grant I et al (2006). Cognitive deficits and degeneration of interneurons in HIV+ methamphetamine users. Neurology 67: 1486-1489.

Chang L, Ernst T, Speck O, Grob CS (2005). Additive effects of HIV and chronic methamphetamine use on brain metabolite abnormalities. Am J Psychiatry 162: 361-369. 
Chompre G, Cruz E, Maldonado L, Rivera-Amill V, Porter JT, Noel RJ (2013). Astrocytic expression of HIV-1 Nef impairs spatial and recognition memory. Neurobiol Dis 49: 128-136.

D'Hooge R, Franck F, Mucke L, De Deyn PP (1999). Age-related behavioural deficits in transgenic mice expressing the HIV-1 coat protein gp120. Eur J Neurosci 11: 4398-4402.

Der-Avakian A, D'Souza MS, Pizzagalli DA, Markou A (2013). Assessment of reward responsiveness in the response bias probabilistic reward task in rats: implications for cross-species translational research. Transl Psychiatry 3: e297.

Eddleston M, Mucke L (1993). Molecular profile of reactive astrocytes: implications for their role in neurological disease. Neuroscience 54: 15-36.

Fernandes SP, Edwards TM, Ng KT, Robinson SR (2007). HIV-1 protein gp120 rapidly impairs memory in chicks by interrupting the glutamate-glutamine cycle. Neurobiol Learn Mem 87: 1-8.

Gupta S, Bousman CA, Chana G, Cherner M, Heaton RK, Deutsch $\mathrm{R}$ et al (2011). Dopamine receptor D3 genetic polymorphism (rs6280TC) is associated with rates of cognitive impairment in methamphetamine-dependent men with HIV: preliminary findings. J Neurovirol 17: 239-247.

Hart CL, Marvin CB, Silver R, Smith EE (2012). Is cognitive functioning impaired in methamphetamine users? a critical review. Neuropsychopharmacology 37: 586-608.

Heaton RK, Clifford DB, Franklin DRJ, Woods SP, Ake C, Vaida F et al (2010). HIV-associated neurocognitive disorders persist in the era of potent antiretroviral therapy: CHARTER Study. Neurology 75: 2087-2096.

Heaton RK, Franklin DR, Ellis RJ, McCutchan JA, Letendre SL, LeBlanc $S$ et al (2011). HIV-associated neurocognitive disorders before and during the era of combination antiretroviral therapy: differences in rates, nature, and predictors. J Neurovirol 17: 3-16.

Heaton RK, Grant I, Butters N, White DA, Kirson D, Atkinson JH et al (1995). The HNRC 500: neuropsychology of HIV infection at different disease stages. J Int Neuropsychol Soc 1: 231-251.

Heaton RK, Marcotte TD, Mindt MR, Sadek J, Moore DJ, Bentley H et al (2004). The impact of HIV-associated neuropsychological impairment on everyday functioning. J Int Neuropsychol Soc 10: 317-331.

Henry BL, Geyer MA, Buell M, Perry W, Young JW, Minassian A (2013). Behavioral effects of chronic methamphetamine treatment in HIV-1 gp120 transgenic mice. Behav Brain Res 236: 210-220.

Hestad K, McArthur JH, Dalpan GJ, Selnes OA, Nancesproson TE, Aylward E et al (1993). Regional brain atrophy in HIV-1 infection: association with specific neuropsychological testperformance. Acta Neurol Scand 88: 112-118.

Hoefer MM, Sanchez AB, Maung R, de Rozieres CM, Catalan IC, Dowling CC et al (2014). Combination of methamphetamine and HIV-1 gp120 causes distinct long-term alterations of behavior, gene expression, and injury in the central nervous system. Exp Neurol 263C: 221-234.

Hu SX, Sheng WS, Lokensgard JR, Peterson PK, Rock RB (2009). Preferential sensitivity of human dopaminergic neurons to gp120induced oxidative damage. J Neurovirol 15: 401-410.

Jaeger LB, Nath A (2012). Modeling HIV-associated neurocognitive disorders in mice: new approaches in the changing face of HIV neuropathogenesis. Dis Model Mech 5: 313-322.

Kesby JP, Hubbard DT, Markou A, Semenova S (2014a). Expression of HIV gp120 protein increases sensitivity to the rewarding properties of methamphetamine in mice. Addict Biol 19: 593-605.

Kesby JP, Markou A, Semenova STMARC (2014b). Cognitive deficits associated with combined HIV gp120 expression and chronic methamphetamine exposure in mice. Eur Neuropsychopharm 25: 141-150.

Kieburtz K, Ketonen L, Cox C, Grossman H, Holloway R, Booth H et al (1996). Cognitive performance and regional brain volume in human immunodeficiency virus type 1 infection. Arch Neurol 53: $155-158$.
Kim S, Jeneson A, van der Horst AS, Frascino JC, Hopkins RO, Squire LR (2011). Memory, visual discrimination performance, and the human hippocampus. J Neurosci 31: 2624-2629.

Kumar AM, Fernandez JB, Singer EJ, Commins D, WaldropValverde D, Ownby RL et al (2009). Human immunodeficiency virus type 1 in the central nervous system leads to decreased dopamine in different regions of postmortem human brains. J Neurovirol 15: 257-274.

Langford D, Adame A, Grigorian A, Grant I, McCutchan JA, Ellis RJ et al (2003). Patterns of selective neuronal damage in methamphetamine-user AIDS Patients. J Acquir Immune Defic Syndr 34: 467-474.

Leeson VC, Robbins TW, Matheson E, Hutton SB, Ron MA, Barnes TRE et al (2009). Discrimination learning, reversal, and set-shifting in first-episode schizophrenia: stability over six years and specific associations with medication type and disorganization syndrome. Biol Psychiatry 66: 586-593.

Leon WC, Bruno MA, Allard S, Nader K, Cuello AC (2010). Engagement of the PFC in consolidation and recall of recent spatial memory. Learn Mem 17: 297-305.

Lindl KA, Marks DR, Kolson DL, Jordan-Sciutto KL (2010). HIVassociated neurocognitive disorder: pathogenesis and therapeutic opportunities. J Neuroimmune Pharm 5: 294-309.

Mark KA, Soghomonian JJ, Yamamoto BK (2004). High-dose methamphetamine acutely activates the striatonigral pathway to increase striatal glutamate and mediate long-term dopamine toxicity. J Neurosci 24: 11449-11456.

Marquez C, Mitchell SJ, Hare CB, John M, Klausner JD (2009). Methamphetamine use, sexual activity, patient-provider communication, and medication adherence among HIV-infected patients in care, San Francisco 2004-2006. AIDS Care 21: 575-582.

Marquine MJ, Iudicello JE, Morgan EE, Brown GG, Letendre SL, Ellis RJ et al (2014). "Frontal systems" behaviors in comorbid human immunodeficiency virus infection and methamphetamine dependency. Psychiatry Res 215: 208-216.

Norman MA, Moore DJ, Taylor M, Franklin D, Cysique L, Ake C et al (2011). Demographically corrected norms for African Americans and Caucasians on the Hopkins Verbal Learning Test-Revised, Brief Visuospatial Memory Test-Revised, Stroop Color and Word Test, and Wisconsin Card Sorting Test 64-Card Version. J Clin Exp Neuropsychol 33: 793-804.

Panenka WJ, Procyshyn RM, Lecomte T, MacEwan GW, Flynn SW, Honer WG et al (2013). Methamphetamine use: a comprehensive review of molecular, preclinical and clinical findings. Drug Alcohol Depen 129: 167-179.

Purohit V, Rapaka R, Shurtleff D (2011). Drugs of abuse, dopamine, and HIV-associated neurocognitive disorders/HIV-associated dementia. Mol Neurobiol 44: 102-110.

Rippeth JD, Heaton RK, Carey CL, Marcotte TD, Moore DJ, Gonzalez R et al (2004). Methamphetamine dependence increases risk of neuropsychological impairment in HIV infected persons. $J$ Int Neuropsychol Soc 10: 1-14.

Roberts AJ, Maung R, Sejbuk NE, Ake C, Kaul M (2010). Alteration of methamphetamine-induced stereotypic behaviour in transgenic mice expressing HIV-1 envelope protein gp120. J Neurosci Methods 186: 222-225.

Santosuosso M, Righi E, Lindstrom V, Leblanc PR, Poznansky MC (2009). HIV-1 envelope protein gp120 is present at high concentrations in secondary lymphoid organs of individuals with chronic HIV-1 infection. J Infect Dis 200: 1050-1053.

Scott JC, Woods SP, Matt GE, Meyer RA, Heaton RK, Atkinson JH et al (2007). Neurocognitive effects of methamphetamine: a critical review and meta-analysis. Neuropsychol Rev 17: 275-297.

Segal DS, Kuczenski R (1997). An escalating dose "binge" model of amphetamine psychosis: behavioral and neurochemical characteristics. J Neurosci 17: 2551-2566. 
Steinberg EE, Keiflin R, Boivin JR, Witten IB, Deisseroth K, Janak PH (2013). A causal link between prediction errors, dopamine neurons and learning. Nat Neurosci. 16: 966-973.

Toggas SM, Masliah E, Rockenstein EM, Rall GF, Abraham CR, Mucke L (1994). Central nervous system damage produced by expression of the HIV-1 coat protein gp120 in transgenic mice. Nature 367: 188-193.

Turchan J, Anderson C, Hauser KF, Sun QM, Zhang JY, Liu Y et al (2001). Estrogen protects against the synergistic toxicity by HIV proteins, methamphetamine and cocaine. BMC Neurosci 2: 3

Vitkovic L, daCunha A (1995). Role for astrocytosis in HIV-1associated dementia. In: Oldstone MBA, Vitkovic L (eds). HIV and Dementia. Springer-Verlag: Berlin. Vol 202, pp 105-116.

Weber E, Blackstone K, Woods SP (2013). Cognitive neurorehabilitation of HIV-associated neurocognitive disorders: a qualitative review and call to action. Neuropsychol Rev 23: 81-98.
Woods SP, Scott JC, Fields JA, Poquette A, Troster AI (2008). Executive dysfunction and neuropsychiatric symptoms predict lower health status in essential tremor. Cogn Behav Neurol 21: 28-33.

Young JW, Geyer MA, Rissling AJ, Sharp RF, Eyler LT, Asgaard GL et al (2013). Reverse translation of the rodent 5C-CPT reveals that the impaired attention of people with schizophrenia is similar to scopolamine-induced deficits in mice. Transl Psychiatry 3: e324.

Young JW, Minassian A, Paulus MP, Geyer MA, Perry W (2007). A reverse-translational approach to bipolar disorder: rodent and human studies in the Behavioral Pattern Monitor. Neurosci Biobehav Rev 31: 882-896.

Young JW, Powell SB, Geyer MA, Jeste DV, Risbrough VB (2010). The mouse attentional-set-shifting task: a method for assaying successful cognitive aging? Cogn Affect Behav Neurosci 10: 243-251.

Supplementary Information accompanies the paper on the Neuropsychopharmacology website (http://www.nature.com/npp) 